\title{
INGRESO Y PRESENCIA DE LAS MUJERES \\ EN LA MATRÍCULA UNIVERSITARIA EN MÉXICO*
}

\author{
Enrolment and continuance of women in mexican universities
}

\author{
Rosa María Huerta Mata**
}

\section{RESUMEN}

Existieron condiciones sociales, culturales, económicas y políticas que confluyeron para el ingreso de las mujeres a las universidades; así como acciones de valentía que mostraron algunas de las primeras estudiantes de educación superior en México. Específicamente en la Universidad Autónoma de San Luis Potosí (UASLP), éstos fueron importantes antecedentes del trabajo actual en materia de equidad de género en esta institución. El análisis que aquí se realiza favorece la construcción del conocimiento sobre la limitada exploración que se ha hecho respecto de las contribuciones que las estudiantes han tenido en la historia de los sistemas de educación superior y en las relaciones de género establecidas por su presencia en la matrícula universitaria.

Palabras Clave: Género en la historia, eduCación superior, estudiantes Universitarias, EQUIDAD DE GÉNERO.

\section{Abstract}

There were social, cultural, economic and political conditions that converged for women to be admitted in universities. In addition to that there were acts of bravery shown by some of the first female students of higher education in Mexico. Particularly at Universidad Autónoma de San Luis Potosí (UASLP), these were important backgrounds of what is being currently worked on gender equity at this institution. The analysis being performed favors the construction of knowledge, over the limited exploration that has been done, in relation to contributions that students have had in the history of university education systems and in gender relations established by its presence in college enrollment.

KEYWORDS: GENDER IN HISTORY, HIGHER EDUCATION, UNIVERSITY FEMALE STUDENTS, GENDER EQUITY.

Recepción: 11 de enero de 2016.

Dictamen 1: 28 de enero de 2016.

Dictamen 2: 23 de febrero de 2016.

Dictamen 3: 21 de julio de 2016.

* Este trabajo se desprende de la tesis doctoral de la autora, titulada Joven, mamá y estudiante: Identidad materna universitaria de 'la madre soltera'.

** Universidad Autónoma del Estado de Hidalgo. Escuela Superior de Actopan.Correoelectrónico:huertamx@ hotmail.com. 
Para comprender la composición de género actual en la matrícula estudiantil en el nivel de licenciatura en la Universidad Autónoma de San Luis Potosí (UASLP), es necesario considerar el proceso histórico en el que se ha configurado tal composición. Este artículo tiene el objetivo de analizar los diversos elementos contextuales e individuales que han influido en el ingreso de las mexicanas a la universidad, así como la forma en que su presencia ha generado cambios sociales y culturales. El trabajo se organiza de la siguiente forma: presento un análisis histórico (de finales del siglo XIX a finales del siglo XX) sobre las primeras universitarias mexicanas. Incorporo los datos relativos al incremento de la matrícula escolar femenina en la educación superior durante el siglo XX. Para el caso específico de la UASLP, expongo información sobre las primeras mujeres tituladas. Por medio de la metodología de relato de vida, presento el análisis de la entrevista realizada a Ma. Cristina Saavedra Maldonado, una de las primeras médicas egresadas de la Universidad Autónoma de San Luis Potosí.

En México, a partir de los años setenta del siglo XX se iniciaron esfuerzos en la promoción de los derechos de las mujeres al denunciarse las condiciones jurídicas y sociales de trato diferencial con respecto de los hombres. De esas primeras acciones se desprendió, en las siguientes décadas, una serie de acuerdos internacionales, ${ }^{1}$ en razón de los cuales México se comprometió a establecer políticas para la promoción de la equidad de género. En 2003, la Asociación Nacional de Universidades e Instituciones de Educación Superior (ANUIES) creó una Red Nacional de Enlaces Académicos de Género, que se derivó posteriormente en Redes Regionales.

En 2009 se celebró la Reunión Nacional de Universidades Públicas "Caminos para la Equidad de Género en las Instituciones de Educación Superior”, la cual se planteó como objetivo trabajar la perspectiva de género como eje transversal, y se propuso una legislación para permitir la creación de comisiones de género dentro de las instituciones de educación superior (IES), a fin de regular la aplicación de un reglamento de equidad dentro de las universidades. Para ello, se sugirió la asignación de recursos anuales por parte de la ANUIES a dichas instituciones (Universidad de Colima, 2011). En la actualidad, la Universidad Autónoma de San Luis Potosí pertenece a la red de Estudios de Género de la Región Noreste,

\footnotetext{
1 "La Cumbre Mundial sobre la Mujer (Beijing, 1995), Los Objetivos de Desarrollo del Milenio (2002) y, más recientemente, la X Conferencia Regional sobre la Mujer de América Latina y el Caribe (CEPAL, Quito, 2007) o la XVII Cumbre Iberoamericana (Santiago de Chile, 2007). Todos los países que firmaron dichos acuerdos se comprometen a aplicar la perspectiva de género en sus políticas públicas, incluidas las políticas educativas" (Alberti, 2009,p. 34). México, al formar parte de estos acuerdos internacionales, en 2006 publicó la Ley General para la Igualdad entre Mujeres y Hombres (Diario Oficial de la Federación, 2006).
} 
junto con las universidades autónomas de Coahuila, Durango, Nuevo León, Tamaulipas y Zacatecas.

En esta dinámica se establecen observatorios de equidad de género dentro de las instituciones de educación superior y se busca implementar el trabajo trasversal sobre la perspectiva de género. Todos estos esfuerzos pretenden que las universidades sean espacios clave en el contexto educativo, donde no sólo se realicen los aprendizajes y se transmitan los conocimientos en la materia, sino además se fomente el respeto a las diferencias y se promueva la igualdad de oportunidades de desarrollo para los miembros que forman parte de estas instituciones, pues se busca alcanzar una cultura democrática. Lo anterior, en razón del bagaje creado por el esfuerzo de algunas académicas feministas que en décadas pasadas comenzaron la elaboración de estudios sobre la disciplina - lo que dio origen a cursos, talleres, programas y departamentos de investigación feministas - (Bartra, 2001; Lagarde, 2003; Urrutia, 2005). Todos estos esfuerzos constantes sobre feminismo, de forma coyuntural con los acuerdos internacionales de género, han permitido detectar y denunciar que en las universidades existen deficiencias en las condiciones de igualdad entre hombres y mujeres.

En este contexto, es fundamental identificar los procesos históricos que han permitido el emprendimiento de políticas de equidad de género dentro de los sistemas de educación superior mexicanos para comprender, más allá de los acuerdos internacionales y las políticas públicas, las luchas históricas y locales de las mujeres para lograr su inclusión en la educación superior. Joan W. Scott (2000, p. 290) señala la importancia de crear conocimientos históricos que posibiliten "romper la noción de fijeza” en las representaciones de género. En este sentido, las mujeres no han cumplido en su totalidad con los mandatos de género que las identifican como personas pasivas, sino que han generado cambios en las estructuras institucionales; tal es el caso de las primeras estudiantes universitarias que establecieron nuevos modelos de participación de las mujeres en la vida pública.

Es fundamental partir de la historia de las mujeres en la universidad para comprender el desarrollo de las dinámicas sobre la distribución de esta población estudiantil. Son escasos los estudios de la historia de las universidades en cuanto a género, y los que existen no se concentran en alumnas, lo que puede significar una limitante en la construcción de este conocimiento. Asimismo, las instituciones de educación superior en el país desde hace tiempo han centrado su interés en las trayectorias de vidas de docentes e investigadoras (Arciga Zavala, 2009; Chávez et al., 2009; García Guevara, 2004; Lara, 2009; López y Félix, 2010; Morley, 1999; Preciado, 2006). 
Es un reto visibilizar las aportaciones de las estudiantes en la conformación histórica de estos sistemas.

\section{EL INGRESO DE LAS MUJERES A LA EDUCACIÓN SUPERIOR EN MÉXICO}

Durante periodos largos, la instrucción académica se consideró innecesaria para la función social que desarrollaban las mujeres, y sólo se les preparaba para las actividades domésticas; de esta manera, su desarrollo se confinó al ámbito familiar. En el porfiriato, las que buscaron una mayor participación en la esfera pública se limitaron a actividades de servicio a los otros. Trabajos que requieren mínima capacitación, en general poco remunerados, y con escasas posibilidades de promoción o superación. Se dedicaban a oficios como obreras o dependientas en tiendas. Mílada Bazant (1999, p. 119) señala que las alternativas laborales de las mujeres de clase baja se restringían a sirvientas, y las de clase media, costureras. Fue a través de la educación en las escuelas de Artes y Oficios como comenzaron a adquirir conocimientos que les permitieron laborar como secretarias en industrias, oficinas públicas y comercios.

En ese mismo periodo encontramos el antecedente del ingreso y la formación de las mexicanas en la educación superior, con la creación de la Escuela Normal de Maestras en el Distrito Federal, en $1890 .^{2}$ Debido a la gran demanda que fue adquiriendo esta institución, a cinco años de su creación presentó problemas de sobrecupo, por esta razón debió cerrar durante un ciclo escolar (Cano, 2000, p. 207). En pocos años, hubo un ingreso masivo de las mujeres al magisterio, lo que confirma su gran interés por acceder a una formación académica más amplia, y a partir de sus conocimientos obtener trabajos con mayor ingreso económico y estatus social.

Otra acción fundamental que dio paso a la presencia de las mujeres en la Universidad fue el acceso de éstas a la escuela Nacional Preparatoria en 1893, aunque años atrás, en 1885 , varias jóvenes ya habían solicitado su ingreso, que les fue negado (Cano, 2000, p. 234). De este modo, algunas demandarían el acceso a espacios que les permitieran alcanzar un nivel educativo superior y distintos a los lugares destinados socialmente para ellas en ese tiempo. La inquietud intelectual manifestada por varias jóvenes mexicanas es indicativo de la generación de cambios 
en cuanto a su posición frente al conocimiento. Aunque cabe aclarar que en esa época, tanto en Estados Unidos como en algunos países de Europa, las mujeres ya habían comenzado a acceder a la educación superior.

En México y en América Latina, la primera mujer titulada en la universidad fue la dentista Margarita Chorné y Salazar, en 1886, profesión que aprendió de su padre, y esto avaló su conocimiento para brindarle el título (Rivera, 2012). ${ }^{3}$ En México, la primera mujer que ingresó como estudiante en la universidad y se tituló es la médica Matilde Montoya, quien, con apoyo del general Porfirio Díaz, logró que la aceptaran en la Escuela Nacional de Medicina, cuyas autoridades la habían rechazado por ser mujer. Al titularse, le fue negada la presentación de su examen de grado. Ante esto, el general Porfirio Díaz intervino emitiendo un decreto para que pudiera graduarse; además confirmó su apoyo al asistir, en compañía de su esposa, al examen recepcional de Matilde Montoya, el 24 de agosto de 1887 (Asociación Nacional de Médicas Mexicanas, 2013). Es relevante que las mexicanas no ingresaron a las aulas universitarias hasta 1882, veintitrés años después de que esto sucediera en Europa. ${ }^{4}$ Lo anterior indica el profundo antecedente en México como sociedad patriarcal, que sigue manteniendo cierta influencia en los sistemas universitarios de este país.

La primera abogada titulada en México fue María Asunción Sandoval Zarco, en julio de 1898 (Alvarado y Becerril, 2002). Todos estos casos son extraordinarios para la época, pues en el porfiriato, el Estado apenas había generado una especie de educación técnica para las mujeres de clase baja, con el objetivo de integrarlas a la industria. ${ }^{5}$ La otra opción de estudio era el magisterio. Socialmente no era aceptado que una mujer optara por una carrera liberal como derecho o medicina; en todo caso, llegaba a estudiar para partera.

La primera ingeniera en nuestro país fue Concepción Mendizabal, en 1930 (Escamilla y Pineda, 2012). ${ }^{6}$ Éste es otro ejemplo — como lo mencionamos en el caso de Margarita Chorné Salazar- de las primeras mujeres universitarias que eligieron su carrera influidas por la profesión de su padre. Mendizabal causa

\footnotetext{
3 "Procedía de una familia de orfebres y dentistas. Su abuelo fue el joyero oficial de la Catedral de México, oficio que continuó su padre durante un tiempo hasta que se dedicó de lleno a la profesión dental” (Rivera, 2012).

4 A partir de 1859, en San Petersburgo, Rusia, se aceptó el ingreso de las mujeres a la escuela de medicina (Palermo, 2006, p. 13).

5 Durante el porfiriato, en 1871, se creó, en la ciudad de México, la Escuela de Artes y Oficios para Mujeres, y similares en otras ciudades del país (Alvarado, 2004, p. 295).

6 Su padre,Joaquín Mendizábal Tamborrel, egresó de la Escuela Nacional de Ingeniería, obtuvo los títulos de ingeniero topógrafo e hidromensor, y el de ingeniero geógrafo en 1883; además, ejerció diversos cargos importantes en su profesión (Escamilla y Pineda, 2012).
} 
asombro para su época, sobre todo porque se formó en una profesión considerada exclusiva para hombres; rompió esquemas en un tiempo en el que lo común para las mujeres que deseaban estudiar era acceder a una de las varias escuelas de educación técnica que los gobiernos posrevolucionarios crearon a partir de los años veinte. En ese tiempo, el Estado, a través de la Secretaría de Educación Pública (SEP), buscaba cambiar las antiguas escuelas de Artes y Oficios por escuelas industriales (Rodríguez, 2002), ${ }^{7}$ donde se fomentaba una educación doméstica con la finalidad de formar amas de casa que contribuyeran, desde el hogar, al desarrollo del país con sus labores de crianza.

Por lo tanto, las primeras mujeres que ingresaron a los sistemas universitarios fueron vistas como extrañas e intrusas, pues irrumpieron en un escenario contemplado como estrictamente masculino. Debió representar para ellas la decisión de tomar riesgos de exclusión social y verse sujetas a situaciones de hostilidad y rechazo, tanto de hombres como de mujeres. La voluntad y la lucha personal de aquellas que se atrevieron a ir en contra de lo establecido socialmente para su género son factores que hicieron posible el ingreso de las mexicanas al sistema de educación superior.

En cambio, la incursión de las mujeres en la formación como maestras normalistas fue vista como adecuada por el Estado posrevolucionario, que tenía el objetivo de brindar a la población una educación que sirviera de base al desarrollo productivo del país. Esto configuró el preámbulo de la incorporación de las mexicanas a la universidad, incluso varias de las primeras universitarias tuvieron como formación inicial el magisterio y posteriormente revalidarían algunas asignaturas para estudiar una licenciatura. Tal fue el caso de la primera abogada en San Luis Potosí, Dolores Arriaga, y la también abogada Socorro Blanc Ruiz, así como Columba Rivera y Antonia Ursúa, respectivamente, la segunda y quinta médicas tituladas en el país.

Entre las vicisitudes de la vida estudiantil que quizá influyeron en las estudiantes de la época, en San Luis Potosí se presentó una situación particular en el periodo de 1913 a 1914, cuando la Escuela Normal fue dirigida y establecida en las instalaciones del entonces Instituto Científico y Literario. ${ }^{8}$ Nos preguntamos si es posible que

\footnotetext{
7 Entre estas escuelas técnicas se encontraban la escuela industrial Gabriela Mistral, la de Artes y Oficios para Señoritas, la Industrialy Doméstica Sor Juana Inés de la Cruz. A las alumnas se les enseñaba economía doméstica, curtiduría de flores, pintura, bordado, lencería, cocina y repostería (Rodríguez, 2002).

8 Como consecuencia del tiempo revolucionario, el 13 de enero de 1913 "se fusionan en el Instituto Científico y Literario las escuelas Normales - de profesores y de profesoras-, o sea que con los tres planteles se formó uno solo. La consecuencia fue que disminuyó tanto el número de alumnos normalistas, que un año después, a mediados de 1914, sólo contaba con ocho estudiantes para profesores" (Rivera y Montejano y Aguiñaga, 1998, p. 36; Benemérita y Centenaria Escuela Normal del Estado de San Luis Potosí, 2013).
} 
algunas de las alumnas normalistas de ese entonces, ante la influencia del ambiente universitario que compartieron, decidieron estudiar como segunda formación una carrera de educación superior.

Asimismo, las maestras normalistas posrevolucionarias tuvieron acceso a un nuevo estatus social al participar, algunas de ellas, en la Revolución de 1910, asistir al primer Congreso Feminista de Yucatán, en 1916, apoyar la Constitución de 1917 y al plan educativo de José Vasconcelos. Son las que a través de sus acciones políticas en apoyo a la cultura de las mexicanas lograron conformar una base para la inserción de las jóvenes en la educación superior (Hierro, 2007, p. 80). De manera que no sólo fueron los esfuerzos individuales de cada una de las primeras universitarias, sino también las acciones grupales de algunas normalistas en el espacio social y político del país, lo que favoreció la presencia de estudiantes universitarias.

Otro elemento que influyó en el ingreso de las mujeres a la universidad son las familias de donde provenían. La clase de élite en el porfiriato, y aun avanzado el siglo XX, no veía necesario el ingreso de sus miembros a los estudios universitarios (Kicsa,1982; Lomnitz,1993), lo que es indicativo de que muchos de los profesionistas de ese entonces no fueron precisamente los ricos. La fuerte vinculación laboral de la clase media alta con la clase alta, y el predominio del desarrollo de una ideología liberal en la primera, posiblemente fue conformando la tolerancia para la inserción de las mujeres como estudiantes universitarias. ${ }^{9}$

Por eso fue común que las primeras mujeres que ingresaron a la universidad contaran con el apoyo de sus familias, las cuales tenían un pensamiento liberal que encerraba ideas de igualdad en consideración a la educación, aunque por lo general sus objetivos no eran precisamente los de la realización profesional de las mujeres, sino la formación de un bagaje cultural que transmitirían a su descendencia. O el pretender respaldarlas con una educación universitaria, como un recurso para defenderse en la vida, sobre todo ante la carencia de la figura paterna. En este sentido, encontramos un aspecto común entre las primeras universitarias: varias de ellas fueron huérfanas de padre a edad temprana. ${ }^{10}$ Estas mujeres trabajaron

\footnotetext{
9 Lomnitz (1993) expone el caso de una familia de élite que mantiene un sistema patriarcal (1820-1980), donde por cuatro generaciones ni los hombres ni las mujeres realizaron estudios universitarios, pues pensaban que éstos eran para la clase trabajadora; ellos se dedicaban al negocio familiar desde muy jóvenes y las mujeres al cuidado del esposo y los hijos. Dadas las condiciones contextuales de finales del siglo XX, posiblemente la apertura de mercados exigió que las mujeres de esta familia comenzaran a ingresar en la educación superior. Esta situación se encuentra generalizada en nuestros días en la élite mexicana.

10 Matilde Montoya, huérfana de padre a los 13 años (Asociación Nacional de Médicas Mexicanas, 2014). Rosario Oyárzun Romero, una de las primeras abogadas en San Luis Potosí, huérfana de padre a los tres años. Sufragó su carrera con trabajos como vendedora de libros o impartía clases (Peñalosa, 2000). Beatriz Velázquez Castillo, de
} 
para pagar sus estudios o, como mencionan Alvarado y Becerril (2002) en el caso de Matilde Montoya, fueron apoyadas por varios benefactores. ${ }^{11}$

Los casos aislados de las primeras mexicanas que ingresaron a la educación superior sentaron las bases para la integración de mujeres en la universidad. Sin embargo, el aumento del número de mujeres matriculadas en las universidades públicas mexicanas se convirtió un proceso lento. Fue a partir de las décadas intermedias del siglo XX cuando comenzó a ser un poco más notoria la prevalencia de estudiantes universitarias.

\section{Presencia de las MUjeres en la UniVERSidad (1940-1970)}

Si bien las primeras universitarias establecieron una lucha particular y llena de valentía para ingresar y permanecer en la universidad, y más tarde ejercer sus profesiones, existió, especialmente de 1940 a 1970, un momento coyuntural en el cual el contexto educativo sufrió un cambio que hizo posible que las mexicanas aprovecharan la oportunidad de ingresar de manera más amplia a la educación superior. En los años cuarenta del siglo XX, en especial después de la Segunda Guerra Mundial, en México comenzó un incremento en el desarrollo industrial, que generó acciones de apoyo por parte del gobierno hacia la educación universitaria, contemplada como un medio para dar instrucción más especializada a la población y estimular el crecimiento económico del país. Asimismo, en esa época empezó a volverse común la práctica — sobre todo en las familias de clase alta y media de padres ilustrados - de brindar educación profesional a las mujeres. Esto se refleja en algunos datos cuantitativos; por ejemplo, en la Facultad de Derecho de la Universidad Nacional Autónoma de México ${ }^{12}$ (UNAM), no fue hasta 1941

1992 a 2000 fue directora de la Facultad de Medicina en la Universidad Autónoma de San Luis Potosí. Egresó de esta institución en 1958. Huérfana de padre a los siete años. Mientras cursaba la preparatoria y toda la carrera de medicina, trabajaba para costear sus estudios (Rodríguez de Palacios, 2001).

11 "Matilde Montoya había arrancado sus estudios médicos en Puebla, pero el presidente Díaz la invitó a finalizarlos en la capital de la república, pues consideraba que nada más justo ni mejor que la primera doctora mexicana se titulara en esta ciudad. Con posterioridad y gracias a su trayectoria académica, contó con el auxilio de Joaquín Baranda, secretario de Justica e Instrucción Pública, a quien ella misma calificara como 'mi bondadoso protector' [...] Por su parte, el gobierno federal le concedió una mensualidad de $\$ 40$ y los gobernadores de Morelos, Hidalgo, Pueblay Oaxaca hicieron lo propio, al señalarle pequeñas pero utilísimas pensiones" (Alvarado y Becerril, 2002).

12 En el libro Historia de la Facultad de Derecho (de la UNAM) se publica una relación del número de mujeres tituladas de 1868 a 1953 (Mendieta, 1976, pp. 364-366). 
cuando hubo titulaciones de mujeres todos los años. En 1947 se alcanzaron 23 acreditaciones de abogadas. ${ }^{13}$

En las estadísticas históricas del Instituto Nacional de Estadística y Geografía (INEGI), la matrícula en educación universitaria en 1930 fue de 23 713, en 1950 pasó a 29892 , y registró un aumento drástico en los años setenta, hasta alcanzar un total de 252200 estudiantes universitarios de ambos sexos (De la Torre, 2002). ${ }^{14}$ Fue así como la Universidad pasó paulatinamente de atender a grupos pequeños conformados por personas que contaban con el privilegio de estudiar a convertirse en un sistema de educación en masa, que se volvió más heterogéneo y complejo al incluir en su matrícula a un mayor número de mujeres.

Durante los años setenta, el entonces presidente Luis Echeverría incrementó la inversión pública en la educación superior, debido a que buscaba elevar el nivel educativo de los mexicanos, como un medio para integrar al país en el desarrollo capitalista. En esta misma década aumentó la migración de las familias del campo hacia zonas urbanas, en busca de mejores condiciones de vida, que pretendían obtener por medio de la educación y el empleo (Izaguirre, 2010, p. 140). Antes del desarrollo del capitalismo, muchas familias pasaron de funcionar como unidades de producción a convertirse en unidades de consumo, y la educación superior era un elemento que facilitaba el acceso a una vida mejor en las ciudades. Las familias de clases medias comenzaron a ver como una inversión la adquisición, por parte de las mujeres, de un trabajo más calificado y mejor remunerado como resultado de una mayor escolarización. Esto contribuyó a que se visualizara a las mujeres, ya no sólo como un apoyo en las labores de servicio hacia sus familiares, sino también como miembros que favorecerían la posición económica y las condiciones de vida de su grupo familiar de origen a través del ejercicio de su profesión.

Sin embargo, esto no se volvió una generalidad. En la mayoría de las ocasiones, las familias permitieron que las mujeres accedieran a la educación superior como un medio de adquisición cultural, o para que la practicaran sólo por unos años y, llegando al matrimonio, renunciaran totalmente al ejercicio profesional, o siguieran ejerciendo su carrera mientras no hubiera hijos a quienes cuidar. Se consideraba la “educación superior como preparatoria para la 'verdadera profesión', el matrimonio (o carrera 'm.t.c.' - mientras te casas-)” (Hierro, 2007, p. 116). Muchas mujeres asumieron el discurso de integrarse a las licenciaturas "femeninas", porque les permitían aplicar los conocimientos adquiridos en su entorno familiar y social. La

\footnotetext{
13 La primera mujer se tituló en 1898. Se titularía otra en 1920. En 1931 hubo dos titulaciones más.

14 Las cifras son totales; no se presentan de manera desagregada el número de mujeres y el número de hombres.
} 
educación universitaria de las mujeres era vista como un patrimonio, un capital cultural, que les serviría en caso de viudez o en cualquier situación que ameritara la aplicación de sus conocimientos en el campo laboral, por si era necesario que trabajaran; como una forma de respaldarlas y no dejarlas sin elementos para defenderse ante la vida.

A pesar de que en el sistema universitario mexicano se reconoce este periodo (1940-1970) como el de mayor incremento de estudiantes universitarios, no fue en estas décadas cuando las mujeres alcanzaron cifras de paridad en la matrícula escolar. ${ }^{15}$ El ingreso de las mujeres a la universidad no desplazó la idea de la maternidad como función primordial de las mexicanas. Las jóvenes de esa época que se incorporaron a la universidad como estudiantes se titularon, pero además ejercieron su profesión (ya sea renunciado al matrimonio y la maternidad, o los incorporaron a su vida profesional); ellas fueron quienes contribuyeron con mayor fuerza al cambio de las expectativas y significados sociales que se tenían sobre las estudiantes universitarias.

\section{HiSTORIA DEL INGRESO DE LAS ESTUDIANTES UNIVERSITARIAS A LA UASLP}

La educación superior en San Luis Potosí data de 1624, cuando era impartida por sacerdotes jesuitas, quienes ocuparon lo que actualmente se conoce como el Edificio Central de la Universidad, hasta su expulsión en 1767. En 1826, en el mismo recinto fue creado el Colegio Guadalupano Josefino, y posteriormente, en 1859, abrió el Instituto Científico y Literario. En 1923 se convirtió en la Universidad de San Luis Potosí ${ }^{16}$ (UASLP, 2000, pp. 6-35).

Entre los antecedentes del impulso para la formación de las potosinas se encuentra la iniciativa llevada adelante en 1906 por el farmacéutico Isidro Palacios, quien impartió en el Hospital Civil y en la Escuela de Artes y Oficios un curso gratuito de farmacia. Tenía el objetivo de crear una carrera para las mujeres (Pedraza, 1979, p. 258). Nos preguntamos si este antecedente de la instrucción técnica de las mujeres

15 La brecha entre el número de alumnas y el número de alumnos presentaría modificaciones más notables a partir de la década de los noventa: "desde 1992 las mujeres representan 43\% de la matrícula total en algunas de las universidades del país. Con diferencias por área, tipo de institución y por nivel” (García Guevara, 2004, pp.103-104).

16 Desde 1934 exhibe el título de Universidad Autónoma de San Luis Potosí. En 1944 por decreto constitucional se reconoce a la Universidad con personalidad jurídica propia. 
en el ámbito de farmacia estimuló el notable ingreso de las potosinas en la carrera de químico farmacéutico, que antecedió a la carrera de químico farmacobiólogo, la cual ha tenido desde sus inicios una mayor población femenina en la UASLP. Cabe aclarar que San Luis Potosí y el Estado de México fueron los únicos que crearon varias escuelas de Artes y Oficios; en San Luis Potosí, para 1908 contaba con 479 alumnas (Bazant, 1999, p. 122). Esto es un fundamento importante respecto de las representaciones que en ese entonces se conformaron en torno a la formación escolar de las mujeres potosinas, reflejo de un pensamiento liberal en cuanto a la educación. ${ }^{17}$

En la UASLP, funcionarios y maestros universitarios hicieron esfuerzos que, aunque de forma aislada, motivaron que la institución fuera un espacio donde se integrara a las mujeres al estudio de las carreras profesionales. Éste es el caso del doctor Juan H. Sánchez — primer rector de la UASLP—, quien inscribió a sus dos hijas en la Universidad (Anguiano, 2003, p. 33). Esta primera acción se fundamenta en la preparación e ideología del entonces rector. Asimismo, el ambiente académico y cultural en el que se desarrollaron sus hijas las involucró de manera más directa en los estudios de educación superior. Todo ello influyó de modo indirecto en la creación de una cultura entre los docentes y funcionarios universitarios. ${ }^{18}$

Otro aspecto a considerar es que varias de las primeras universitarias potosinas estudiaron la preparatoria en la Universidad o en el Instituto Científico y Literario, que la antecedió, situación que pudo establecerse como un ambiente que influyó en su incursión en la formación profesional.

\section{LAS PRIMERAS PROFESIONISTAS TITULADAS EN LA UASLP}

En la historia oficial de algunas Escuelas y Facultades de la UASLP encontramos referencias a sus primeras estudiantes universitarias. Sin embargo, este reconocimiento no es generalizado; por ejemplo, en el libro que narra la historia de la Facultad de Ingeniería no se mencionan a las mujeres que han cursado sus carreras. ${ }^{19}$ Por su parte, la

17 Este pensamiento se manifiesta al ser la Universidad Autónoma de San Luis Potosíla primera en el país en adquirir su autonomía en 1923. El primer rector de la UASLP fue el doctor Juan H. Sánchez (UASLP, 2000, p. 33).

18 Claro ejemplo de esta afirmación es el rector de la Universidad Roberto Leyva Torres (1972-1976), cuya hija, Elisa Leyva Ramos, en 1979 se tituló como química en la UASLP. Por consejo de su padre, ella continuó sus estudios en el extranjero, donde realizó la maestría, el doctorado y el posdoctorado en la Universidad de Ohio, en Estados Unidos (Herrera, 2012, pp. 4-9).

19 Me refiero al libro Facultad de Ingenieria. Pasado y presente de la Facultad de Ingenieria (Facultad de Ingeniería de la UASLP, 1993). 
Facultad de Derecho publicó una relación de egresadas de 1920 a 1975 (fecha en la que termina la lista). ${ }^{20}$ No fue hasta 1965 cuando hubo egresadas cada año. ${ }^{21}$ En los años setenta se incrementó el número de egresadas al doble con respecto de los años anteriores. En 1974 terminaron sus estudios de Jurisprudencia 22 alumnas. ${ }^{22}$

Hay casos que merecen analizarse con detenimiento. En 1916 ingresó la primera mujer a la escuela de Jurisprudencia en San Luis Potosí; su nombre, Dolores Arriaga. Por destacarse como la mejor alumna de su generación, se tituló por el alto promedio de calificaciones que obtuvo en todas las asignaturas que cursó. Más tarde ejercería su profesión trabajando como jueza en varios distritos judiciales, en Rioverde, Ciudad Santos, Cerritos, Salinas, Venado, y en la capital del estado. En 1922 se convirtió en la primera mujer magistrada en el Supremo Tribunal de Justicia, cargo que desempeñó hasta su jubilación en 1967 (Villegas, 2007, p. 202). Le sigue otra mujer destacada entre las primeras egresadas de la escuela de jurisprudencia, Socorro Blanc Ruiz, titulada en 1955. En ese mismo año se convirtió en la primera mujer en México, por lo tanto, en San Luis Potosí, en asumir de forma interina una presidencia municipal en el estado (NOTIMEX, 2009, octubre 23).

Ambos casos son paradójicos. Dolores Arriaga ejerció puestos políticos de alto rango en el país cuando aún no les era permitido el voto a las mexicanas. Fue magistrada por 45 años, de los cuales sólo pudo votar por los representantes políticos durante los últimos 13 años, pues en México, las mujeres no tuvieron acceso al voto hasta 1953. Además, no fue hasta este año cuando ellas pudieron acceder a cargos políticos de elección pública, situación que limitó su desarrollo profesional. El caso de la licenciada Blanc Ruiz es representativo en dicho derecho, pues ella lo ejerció a dos años de que las mexicanas lo obtuvieron, aunque accedió a él de forma interina.

Por su parte, la Escuela de Medicina presenta una relación de las alumnas egresadas de la Facultad de Medicina de 1881 a 1975 (Alcocer, 1976, pp. 82-105). ${ }^{23}$ Fue a partir de 1946 cuando hubo egresadas cada año. El número de titulaciones de mujeres médicas se incrementó a partir de los años setenta del siglo XX. ${ }^{24}$

20 El libro Apuntes para la historia de la Facultad de Derecho de la UASLP dedica un capítulo a "La mujer abogada" (Villegas, 2007,pp. 198-201).

${ }^{21}$ De 1920 a 1968 egresaron de una a cuatro mujeres de la Escuela de Derecho, aunque no se presentaron titulaciones de manera consecutiva cada año. En 1969 fueron cinco las egresadas.

22 En la carrera de Derecho, que antiguamente era predominantemente masculina, se ha incrementado la presencia de mujeres, de modo que en la actualidad la Facultad de Derecho de la UASLP cuenta con una población mayoritariamente femenina. En el ciclo 2013-2014, la población de estudiantes se componía en 51.6 por ciento de alumnas y en 48.4 por ciento de alumnos (UASLP, 2014).

23 En el libro: "Historia de la Escuela de Medicina de la UASLP".

${ }^{24}$ La primera médica fue María Castro, en 1912. Y 1920 se tituló la segunda mujer médica, Cirina Portales. En 1920 solo hubo cinco egresadas. En la década de los treinta egresó una mujer, Ma. del Carmen Bravo. 
Ha existido presencia femenina desde el origen de diversas carreras en la UASLP. La carrera de contador público y auditor inició en 1938. En la generación de 1940 se encontraban, entre sus estudiantes, Josefina Gutiérrez, María del Socorro Méndez Arcaraz y Emma Victoria Torres Mora. ${ }^{25}$ La Facultad de Ciencias Químicas publicó una lista de egresados de 1882 a 1979 (Pedraza, 1979, pp. 59-81) ${ }^{26}$. No fue hasta 1940 cuando se incrementó a 12 el número de tituladas de las diversas carreras que se ofrecían en esta Facultad. A partir de los años setenta aumentó notablemente el número de titulaciones de mujeres: en 1978 hubo 64 tituladas. ${ }^{27}$ Del mismo modo, en la Facultad de Estomatología, el número de tituladas se incrementó en los años setenta del siglo XX (Facultad de Estomatología de la UASLP, 2007, pp. 175-183). Cabe señalar que éstas son las mujeres egresadas, pero a las aulas universitarias acudieron muchas más a estudiar, pero no todas lograron titularse.

En las primeras carreras de las Facultades y Escuelas de Ciencias de la Información, Hábitat, Contaduría y Administración, Ciencias de la Comunicación, Estomatología, Economía, Psicología, Ciencias y Ciencias Químicas hubo mujeres desde las primeras generaciones, y varias de ellas se titularon. ${ }^{28}$ Así, las preferencias profesionales de las universitarias en la UASLP se orientaron de forma diversa, no sólo hacia los ámbitos socioadministrativos y de la salud —como aún se manifiesta en las generaciones actuales-, sino también en ciencias exactas e ingeniería y tecnología — aunque en menor número-. Debemos destacar que en las carreras denominadas "masculinas", como Derecho, se presentó en 1920 la primera mujer titulada, más de un siglo después de la apertura de la carrera en San Luis Potosí, en 1826. De ingeniero civil, ${ }^{29}$ la primera titulación de una mujer fue en 1967 , casi un siglo después del inicio de esta carrera, en 1871. Mientras que de ingeniero topógrafo hidrólogo no se tituló una mujer hasta 1979, más de un siglo después de la apertura de esta opción profesional en San Luis Potosí.

A partir de los años setenta del siglo XX en adelante se concentró un mayor número de primeras mujeres tituladas. Hay ejemplos de que los primeros títulos

25 Es la única mención que hacen de las primeras estudiantes, en el libro Historia de la Facultad de Contaduría y Administración de la UASLP (Reyes, 2003, p. 32).

26 En el libro Apuntes Históricos de la Escuela de Ciencias Quimicas.

27 Laprimera mujer que se tituló fue Esther Cuéllar de Vela Vázquez, como "Ensayador químico" en 1928 (Pedraza, 1979, p. 59).

${ }^{28}$ Con los datos expuestos en el artículo de Delgado (2000), además del apoyo en otras fuentes bibliográficas, elaboramos el siguiente análisis de las alumnas egresadas en los siglos XIX y XX.

29 En la UASLP, como en otras universidades del país, impera un lenguaje androcéntrico en los títulos universitarios. No fue hasta octubre de 2007 cuando la UASLP comenzó a expedir los títulos en femenino (Unidad de Enlace de Trasparencia y Acceso a la Información de la UASLP. Expediente 788/TA15.1/003-2015). 
que se expidieron en las carreras pertenecieron a mujeres, como es el caso de Minie Consuelo Pérez Villegas, quien se tituló de licenciada en administración en 1977; Olivia Trujillo Faz, como licenciada en administración pública en 1986 (Delgado, 2000, p. 9). En la Escuela de Ciencias, Sofía Elizabeth Acosta Ortiz, de electrónico físico en 1982; Cecilia Guadalupe Peña de la Maza, de físico teórico en 1982. Ligia María Ramé Narváez de electrónico en sistemas digitales en $1987^{30}$ (Facultad de Ciencias de la UASLP, 1998, pp. 21, 22, 45). Adriana González Santos fue la primera en titularse, en 1983, de la carrera de Diseño Gráfico (Paláu, 2000, p. 246) (véase el cuadro 1). Cabe destacar que estas últimas se ubican en el ámbito de ciencias y tecnología, cuyas carreras se caracterizan en la actualidad por estar conformadas en más de 60 por ciento por población masculina; por lo tanto, las hace un caso excepcional, pues no sólo son de las primeras mujeres que ingresaron en dichas áreas, sino además son las que obtuvieron los primeros títulos expedidos por estas carreras en la Universidad Autónoma de San Luis Potosí.

El segundo, tercero y cuarto títulos otorgados en la Escuela de Odontología, actualmente Facultad de Estomatología, pertenecieron a Cleotilde Rivera Martínez, en 1951, y a Evelia Ruelas Ornelas y María Bertha Arriaga Serment, en 1952 (Facultad de Estomatología, 2007, p. 175). Además, varias de las primeras mujeres que estudiaron en la UASLP obtuvieron su titulación por el alto promedio de calificaciones al concluir la carrera: la primera médica, María Castro, en 1912 (Delgado, 2000, p. 6); la primera abogada, Dolores Arriaga, en 1920 (Villegas, 2007, p. 202); la primera licenciada en comercio exterior, Verónica Araiza López, en 1993 (Delgado, 2000, p. 12), y la primera electrónica física, Sofía Elizabeth Acosta Ortiz, en 1982 (Facultad de Ciencias de la UASLP, 1998, p. 21).

Todo lo anterior deja claro que algunas de las primeras mujeres universitarias fueron estudiantes destacadas: terminaron sus estudios en tiempo y forma, con resultados sobresalientes. Incluso algunas tuvieron trayectorias de formación continua y superación, como es el caso de la médica María de Jesús Uresti, quien obtuvo dos títulos universitarios: en 1920, el de partera, y en 1929, el de médica (Delgado, 2000, p. 6).

En este análisis histórico observamos que muchas estudiantes potosinas de la Universidad Autónoma han constitudido, a partir de un esfuerzo personal, el precedente del que ahora es reconocido como un objetivo institucional: lograr la equidad de género, al ser las primeras egresadas en carreras que incluso se consideraban 
CUADRO 1. RELACIÓN DE MUJERES QUE OBTIENEN LOS PRIMEROS TÍTULOS Y LA TITULACIÓN POR ALTO PROMEDIO EN LAS CARRERAS DE LA UASLP

\begin{tabular}{llll}
\hline Primeros títulos $\begin{array}{l}\text { Nombre } \\
\text { que se expiden en la } \\
\text { UASLP }\end{array}$ & \multicolumn{1}{c}{ Carrera } & Año \\
\cline { 2 - 4 } & Minie Consuelo Pérez Villegas & Licenciada en administración & 1977 \\
\cline { 2 - 4 } & Sofía Elizabeth Acosta Ortiz & Electrónico Físico & 1982 \\
\cline { 2 - 4 } & Cecilia Guadalupe Peña & Físico Teórico & 1982 \\
\cline { 2 - 4 } & Adriana González Santos & Diseño gráfico & 1983 \\
\cline { 2 - 4 } & Olivia Trujillo Faz & Licenciada en Administración Pública & 1986 \\
\cline { 2 - 4 } $\begin{array}{l}\text { Ligia María Ramé Narváez } \\
\text { alto promedio de } \\
\text { calificaciones }\end{array}$ & Electrónico en sistemas digitales & 1987 \\
\cline { 2 - 4 } & Doría Castro & Medicina & 1912 \\
\cline { 2 - 4 } & Sofía Elizabeth Acosta Ortiz & Electrónico Físico & 1982 \\
\cline { 2 - 4 } & Verónica Araiza López & Comercio exterior & 1993 \\
\hline
\end{tabular}

Elaboración propia con datos obtenidos en Delgado, 2000; Facultad de Ciencias UASLP, 1998; Paláu, 2000; Villegas, 2007.

socialmente exclusivas para hombres. Destacaron como estudiantes y después fueron, varias de ellas en el nivel nacional, las primeras mujeres en ocupar cargos administrativos en las universidades o en instancias gubernamentales. Algunas fueron miembros fundadoras de asociaciones femeninas, como la médica María Castro, quien creó la Asociación de Médicas Mexicanas (Herrera, 2011, p. 9). La también médica María Cristina Saavedra Maldonado fundó la Asociación Estatal de Médicas Potosinas. Ambas organizaciones son integradas por mujeres que buscan generar una representación colectiva en los ámbitos del ejercicio profesional.

No fue sencillo para ellas, pues al generalizar su presencia en las universidades fracturaron el binomio que adjudicaba las capacidades racionales para los hombres y lo intuitivo para las mujeres. Ello generó modificaciones sobre el orden de género al integrar a su identidad el ser universitarias. Scott refiere que "'hombre' y 'mujer' son al mismo tiempo categorías vacías y rebosantes. Vacías porque carecen de un significado último, trascendente. Rebosantes, porque cuando parecen estables, contienen en su seno definiciones alternativas, negadas o eliminadas" (Scott, 2000, p. 301). Las condiciones históricas y las acciones de las mujeres dan paso a una reconstrucción permanente de las representaciones y los marcos de acción sobre los espacios que han obtenido o pretenden alcanzar en el mundo académico y profesional. Considerando lo anterior como un proceso discontinuo, muchas veces con limitantes y conflictos, planteamos que es relevante elaborar el relato de 
vida de una de las primeras universitarias potosinas, a fin de recuperar la voz y la memoria acerca de las dinámicas sociales en las que se desarrolló su integración y formación universitaria.

\section{RELATO DE VIDA UNIVERSITARIA: \\ la médica María Cristina SaAvedra Maldonado}

Las primeras estudiantes universitarias fueron consideradas como una amenaza al equilibrio establecido socialmente en las relaciones entre hombres y mujeres; su presencia en la universidad fue cambiando algunas de las representaciones referentes a su inclusión en el campo académico. La médica María Cristina Saavedra Maldonado ${ }^{31}$ es una de las primeras mujeres que estudió en la Escuela de Medicina de la UASLP, en la generación 1944-1950. Aparece en la relación de alumnas tituladas de esta Escuela, en el lugar número veintidós (Alcocer, 1976, p. 88).

Cursó la educación primaria en la escuela para señoritas Mariano Arista. ${ }^{32}$ Quien fue su maestra en sexto año de primaria, por contar María Cristina con un buen promedio de calificaciones, la motivó a continuar estudiando. Sin embargo, las opciones que tenía para asistir a la escuela secundaria en la ciudad sólo eran los colegios particulares. "La maestra dijo: tienes que seguir estudiando, yo voy a hablar con el director de la Universidad, y hay becas" (médica Saavedra Maldonado. P2: 2, 3:3). ${ }^{33}$

La doctora Saavedra obtuvo una beca para estudiar la secundaria en la UASLP, la cual consistió en no pagar la colegiatura; para ello debía conservar el promedio de calificaciones y no reprobar ninguna materia. En ese entonces, la Universidad no tenía ninguna normativa para excluir a las mujeres del acceso a la educación

31 Entrevista personal realizada el 16 de marzo de 2012, en la casa de la doctora, en la ciudad capital de San Luis Potosí. Nació el 29 de septiembre de 1924, en San Luis Potosí, capital. La médica contaba con 87 años de edad al momento de la entrevista. Fue la primera mujer médica familiar jubilada del Instituto Mexicano del Seguro Social (IMSS) en San Luis Potosí, puesto que desempeñó por 27 años, 14 de los cuales fue la única médica en dicha área en el Seguro Social. Fundó la Asociación Estatal de Médicas Potosinas, ahora Colegio de Médicas Potosinas. Realizó la labor de integrar a la entonces Asociación Estatal de Médicas Potosinas a la Asociación de Médicas Mexicanas, ahora Federación de Asociaciones y Colegios de Médicas Mexicanas. En esta última es integrante del Consejo Directivo Nacional, como vicepresidenta normativa. Fue acreedora, en 2005, a la medalla Matilde P. Montoya, máxima condecoración otorgada por la Federación de Asociaciones de Médicas Mexicanas, por destacar en la práctica profesional, la trayectoria personal y social. Tuvo seis hijos.

32 En los años cuarenta, las escuelas públicas mixtas no eran comunes en las zonas urbanas; existían en mayor número en las áreas rurales.

33 La entrevista fue grabada, transcrita y codificada. Llevé a cabo un análisis del nivel conceptual en el programa Atlas.ti., versión 7.1.8. La metodología utilizada es el análisis del contenido del relato de vida de la participante. 
media y superior; tanto ellas como los hombres podían beneficiarse con una beca escolar. ${ }^{34}$ Sin embargo, ingresar a la Universidad le generó conflictos familiares a la doctora Saavedra, pues no era bien visto que una muchacha asistiera a una escuela donde la mayoría de los estudiantes eran hombres. En los años cuarenta del siglo XX, la Universidad Autónoma de San Luis Potosí se ubicaba en lo que ahora se conoce como el Edificio Central de la Universidad, el cual se encuentra frente a la plaza Fundadores, en el centro histórico de la ciudad.

Hasta tenían prohibido las chicas pasar por enfrente [del edificio de la Universidad], había un jardín y mucha gente iba por ahí, pero a las muchachas que pasaban por ahí, pues los muchachos les silbaban, les decían piropos, y decían: no, no pasen por ahí, que les dicen groserías (médica Saavedra Maldonado. P2: 2, 5:5).

Las condiciones sociales representaban el mayor obstáculo para el acceso de las mujeres a la educación superior, más que las cuestiones institucionales. En especial para las clases media y alta, de las cuales provenían varias de las primeras mujeres universitarias, el que asistieran a la Universidad no se convirtió tanto en un problema económico, sino moral. Por esta razón, fue más aceptable que cursaran carreras en las que obtuvieran conocimientos que servirían en sus funciones de crianza al momento de incursionar en la maternidad. Asimismo, no hay que dejar de contemplar que las mujeres también decidían no ingresar a la universidad, a pesar de contar con los elementos para hacerlo, o elegían carreras que les permitían responder a los mandatos de género. Ambas situaciones generaban en ellas satisfacción por apegarse a patrones que les otorgaban reconocimiento social.

A pesar de estas circunstancias, María Cristina deseaba acudir a las aulas universitarias, por lo que sostuvo ante sus padres la decisión de estudiar con el argumento de disponer de una beca, la cual mantuvo durante sus estudios de secundaria, preparatoria y la carrera de medicina. ${ }^{35} \mathrm{Al}$ preguntarle sobre su vocación como médica, responde:

34 En el Estatuto Orgánico de la UASLP de 1943 (consultado en el Centro de Documentación Histórica Lic. Rafael Montejano y Aguiñaga de la UASLP) no se mencionan los requisitos para obtener una beca ni para mantenerla, sólo se aducen los motivos por los cuales podían perderla los estudiantes, entre los que se encuentra realizar actos de orden político o religioso. Es posible que en ese tiempo fuera costumbre otorgarlas por recomendación. En el caso de la doctora Saavedra, debido a su alto desempeño escolar, su profesora de sexto grado de primaria habló con el director de la Universidad y la recomendó para la obtención de una beca en esa institución.

35 De acuerdo con el Censo Nacional Universitario de 1949, la Universidad Autónoma de San Luis Potosí era una de las 19 instituciones de educación superior que existieron en ese tiempo en el país. Tenía 654 alumnos, que se 
¿Sabes en quién me inspiré? [...] Cuando yo era chica, yo veía que la doctora Uresti, ${ }^{36}$ era partera, doctora [...] cuando yo era chica. Ella llegaba a las casas con su maletín, vestida de blanco, en su carretela de caballos, e iba a atender a las casas, a las que tenían sus bebés [...] y entonces yo ya veía que llegaba, ya nacía un bebé y otro bebé, yo la veía muy vestida de blanco, iba con su maletín, se subía a su carretela, y yo pensaba jay yo quisiera ser como ella! (Médica Saavedra Maldonado. P1: 2, 4:4).

El surgimiento de modelos legítimos de mujeres profesionistas sentó el precedente para que otras construyeran expectativas, no solo para la inserción en la universidad, sino además para la obtención de un título y el consecuente ejercicio de la profesión. La vinculación de la labor profesional de las primeras universitarias potosinas, en la vida cotidiana de las más jóvenes, originó un efecto inmediato sobre su propio género. Esto se convirtió en un eje fundamental del cambio cultural en la formación profesional de las potosinas. ${ }^{37} \mathrm{Sin}$ embargo, ese modelo no fue compartido de forma integral en la sociedad. Por ejemplo, cuando la doctora Saavedra expresó en su familia la decisión de estudiar medicina, las reacciones fueron diversas: "Mi mamá era la que no quería, porque decía 'esa muchacha se va a echar a perder en esa escuela con tanto hombre' [...]” (médica Saavedra Maldonado. P1:1, 2:2). San Luis era una sociedad conservadora, y posiblemente con lo que más tuvieron que luchar las mujeres que deseaban estudiar fue con los prejuicios sociales de esa época, pues no era común que la Universidad se considerara como un espacio para las jóvenes, mucho menos contemplar su presencia en una carrera como medicina donde predominaban los hombres, y además se estudia la anatomía humana, lo cual acentuaba la idea de arriesgar el destino moral de estas mujeres.

En los años cuarenta, en México se pretendía generar una educación que fortaleciera el desarrollo económico del país. En San Luis se incrementó la actividad industrial. El periódico El Heraldo, en una nota publicada en 1948, reconoció a

distribuían en las siguientes opciones educativas: Secundaria, Preparatoria, Enfermería, Comercio y Administración, Jurisprudencia, Ingeniería, Medicina, Química, Odontología. Las otras universidades que contaban con la carrera de medicina eran las de México, Guanajuato, Hidalgo, Jalisco, Michoacán, Nuevo León, Oaxaca y Yucatán (De la Torre, 2002). Otras opciones de estudio en los años cuarenta en San Luis fueron la Normal Mixta y la Escuela de Artes y Oficios para Señoritas.

36 María de Jesús Uresti Leija es la cuarta mujer titulada de la escuela de medicina de la UASLP (Alcocer, 1976,p. 85).

37 En el caso de la medicina, las médicas transgredieron las normas de género al adquirir reconocimiento social - como lo hizo la doctora Uresti en la ciudad de San Luis Potosí- por realizar una trayectoria laboral en un área que generaba prestigio para los hombres y donde era prácticamente inexistente la presencia de las mujeres. 
San Luis Potosí como la tercera ciudad más industrializada de la República. ${ }^{38}$ Por esta razón, se exigió una mayor especialización de sus trabajadores, lo cual pudo motivar que en algunas familias se brindara más importancia a la idea de que sus hijos e hijas asistieran a la universidad. "Mi papá decía 'eso te gusta, eso quieres, pues a ver si puedes..., y no vayas a salir a medio camino, ya no me gustó, me voy a salir, ¡menos! me voy a casar'. Entonces era un reto, ¿̇verdad?” (médica Saavedra Maldonado. P1:4,11:11). En el "a ver si puedes" se encuentra un permiso implícito, no reconocido abiertamente por su padre, situación que se complementó con el deseo de María Cristina de estudiar y, sobre todo, con el desafío que representaba su presencia en la Escuela de Medicina.

La medicina tuvo su origen como una profesión liberal para hombres. Paulatinamente fue aceptada como apta para las mujeres, pues se comenzó a relacionar con las funciones maternas del cuidado, la higiene y la salud de la familia (Palermo, 2006, pp. 14-15). Sin embargo, las primeras universitarias que incursionaron como estudiantes en este campo académico lidiaron con los estereotipos de género, que marcaron su formación y, no en pocos casos, influyeron en su deserción escolar. En el grupo con el que estudió medicina María Cristina, al inicio eran cuatro o cinco mujeres. Los hombres, tanto estudiantes como profesores, buscaban desanimarlas manifestando una abierta disconformidad con relación a que estuvieran cursando la carrera.

Los hombres, los maestros o los alumnos, hacían la guerra contra las mujeres. -Para qué estudias medicina, te vas a casar. Tienen familia, nunca acaban, no sirven para la medicina porque se casan, tienen hijos y nunca terminan. Se enamoran-- Bueno nos decían tanta cosa, entonces muchas lloraban.-Mejor dedícate a ayudarle a tú mamá en la cocina, para que cuando te cases, pues sepas hacer algo. Aprende costura, corte y confección, cocina-. Entonces muchas, pues... no sé, ya no les gustaría, o no les gustó el ambiente, y se salían. Entonces, pues me salió la valentía, no sé de dónde, pero yo seguí estudiando los seis años y fui la única que terminó.

A mí si me decían... pues no sé, se me resbalaría todo, porque nunca anduve ni que llorando, ni que ya no vengo. Los maestros algunos eran los que trataban mal a las mujeres, pero los compañeros en sínunca querían mujeres (médica Saavedra Maldonado. P1:3, 6:8).

38 La industria potosina sí concurrirá a la exposición de la recuperación: No podía ser de otro modo, ya que San Luis es la tercera ciudad industrial de la República ("La industria potosina", 1948:6). Consulta realizada en la hemeroteca potosina. 
Lo anterior refleja que la presencia de las primeras estudiantes en la Escuela de Medicina de la UASLP creó situaciones de violencia y exclusión ante la amenaza de un menor control del orden patriarcal sobre las mujeres. Además, es posible que los hombres hayan sentido temor de ser evidenciados ante sus compañeros como superados por las mujeres en los aspectos intelectuales, que se atribuían de dominio meramente masculino. Fue el ingreso de ellas al espacio público y el acceso a contextos de conocimiento en educación superior lo que representó, no sólo una competencia académica para los estudiantes, sino también un potencial desafío laboral.

La división sexual del trabajo asigna como primordiales las funciones reproductivas de las mujeres; es así como se naturaliza que deben ocupar el espacio privado. De este modo, sus labores cobran menor importancia social y se limitan sus capacidades de decisión y acción en el espacio público. Este último, desde la cultura heteropatriarcal, es el que se destina a los hombres; en razón a ello, sus labores tienen un mayor reconocimiento social y económico; por lo tanto, les otorga más poder y ventaja para la adquisición de posiciones de igualdad.

Silvia Bolos (2008, pp. 17-18) señala que en la modernidad se demarca con mayor fuerza la división de estos espacios sociales para los hombres y las mujeres. Fue a partir de que comenzó la adquisición del reconocimiento como ciudadanas y su igualdad ante la ley cuando las mujeres tuvieron una mayor inclusión en el espacio público. La misma autora señala que los grupos feministas cuestionan el hecho de que las mujeres hayan alcanzado la igualdad; establecen que existen diferencias y que es fundamental hacer visibles en el espacio público situaciones del ámbito privado, pues la participación de las mujeres en lo público no ha estado acompañada de condiciones de igualdad.

Lo anterior deja claro que, a pesar de la mayor presencia de las mujeres en lo público, lo privado aún es reconocido en gran medida como idóneo para ellas, y es en donde siguen desarrollando gran número de actividades en su vida diaria. Por eso es importante mostrar las dificultades que afrontan al combinar su presencia en ambas esferas. De este modo, los apoyos y las condiciones que vivan en el espacio privado serán determinantes para que su inclusión en lo público se dé en circunstancias de mayor igualdad. Un claro ejemplo de los antecedentes en esta cuestión es el testimonio de la médica María Cristina, pues refleja las tensiones sociales vividas como resultado de sus acciones al ingresar y permanecer en la UASLP, puntos clave para evidenciar las primigenias labores personales de las mexicanas realizadas al tratar de conciliar su ámbito privado con el emprendimiento de ocupar un lugar como estudiantes universitarias. 
La discriminación hacia las primeras universitarias fue más social que institucional. Al integrarse las mujeres como alumnas en la universidad, la educación superior se convirtió en un lugar de diversidad de género, lo cual propició varias respuestas, ya de tolerancia, ya de violencia, ya ambiguas. De hecho, fue en gran medida un trabajo personal (de cada una de ellas) lo que abrió paso a la creación del espacio educativo que ahora disfrutan las nuevas generaciones de estudiantes universitarias.

\section{Conclusiones}

La integración de las mujeres en la universidad ha sido un proceso largo de lucha y resignificación de los estereotipos sobre la función social más valorada para ellas: la maternidad. Las primeras universitarias hicieron esfuerzos individuales, más que colectivos, para alcanzar su formación profesional.

En el caso particular de San Luis Potosí, varias de las primeras universitarias potosinas se caracterizaron por pertenecer a la clase social media alta; algunas eran hijas de padres liberales, como el primer rector de la Universidad, entre otros funcionarios de la institución; otras fueron huérfanas de padre; asimismo algunas de las primeras universitarias eran de formación inicial normalistas.

Encontramos dos características en el espacio físico - es decir, enel Edificio Central - donde se impartía la formación universitaria en San Luis Potosí, que posiblemente motivaron el ingreso de las mujeres a la educación universitaria. 1) En 1913 compartieron instalaciones el Instituto Científico y Literario (después UASLP) y la Escuela Normal, lo cual tal vez influyó para que algunas normalistas abandonaran sus estudios y se inscribieran en el Instituto Científico y Literario, o, bien, una vez que terminaran la Escuela Normal decidieran estudiar una carrera universitaria. 2) Asimismo, cuando la UASLP se encontraba en el Edificio Central, en ese mismo recinto se impartía la educación secundaria y la preparatoria, hecho que propició que muchas de las que fueron sus estudiantes en estos niveles ingresaran posteriormente a una carrera universitarias.

Observamos dos elementos de heterogeneidad en las dinámicas de ingreso a la UASLP en las primeras estudiantes. Uno es que, al parecer, varias pertenecían a una clase social media alta, pero no fue la norma, pues hubo quienes trabajaron para costear sus estudios, y otras obtuvieron becas escolares. El otro elemento se centra en la diversificación de la presencia de las primeras universitarias tanto en 
las carreras socioadministrativas y de la salud como (en menor número) en ciencias exactas e ingeniería y tecnologías.

En una sociedad como la de San Luis Potosí, caracterizada en el siglo XX por su identificación con principios liberales y, a su vez, regida por una moral conservadora, al parecer, con base en el relato de vida de la médica Saavedra Maldonado ambos aspectos posiblemente ubicaron a muchas de las primeras universitarias potosinas en una situación particular de tensión social, en especial al pretender cursar carreras que se consideraban para hombres, como la de medicina. De manera que la valentía y la determinación en estas mujeres fueron el fundamento de los cambios sociales y culturales necesarios para la presencia de un mayor número de estudiantes en la UASLP.

Asimismo, las primeras médicas potosinas se distinguen por haber consolidado una asociación nacional de médicas (María Castro creó la Asociación de Médicas Mexicanas) y una estatal (María Cristina Saavedra Maldonado fundó la Asociación Estatal de Médicas Potosinas). Además, el ejercicio profesional en la ciudad de mujeres como la doctora Uresti fue consolidando modelos que seguirían las jóvenes y configuró la generación de modificaciones culturales en cuanto a las expectativas en torno a la formación profesional de las potosinas.

Los esfuerzos de las mujeres por pertenecer a la universidad han ido cerrando la brecha de género y han creado cambios significativos en la composición de la matrícula universitaria. No obstante, su integración en la institución de forma equitativa con los hombres, las transformaciones en su identidad y en las representaciones como profesionistas se mantienen como retos permanentes, en los que, además, se entreteje la heterogeneidad de la población de mujeres universitarias.

\section{BIBLIOGRAFÍA}

Alberti, P. (2009). Certificación, calidad y género en las instituciones de educación superior. El caso del Colegio de Postgraduados en Ciencias Agrícolas. En A. Lara (coord.). Género en educación. Temas, avances, retos y perspectivas (pp.33-43). Distrito Federal, México: Universidad Pedagógica Nacional.

Alcocer, A. (1976). Historia de la escuela de Medicina de la UASLP. San Luis Potosí, México: Universidad Autónoma de San Luis Potosí.

Alvarado, L. (2004). La educación "superior" femenina en el México del siglo XIX. Distrito Federal, México: Universidad Nacional Autónoma de México. 
Alvarado, L., y Becerril, E. (2002). Mujeres y educación superior en el México del siglo XIX. En L. E. Galván (coord.). Diccionario de la historia de la educación en México. Distrito Federal, México: Universidad Nacional Autónoma de México, Consejo Nacional de Ciencia y Tecnología, Centro de Investigaciones y Estudios Superiores en Antropología Social. Recuperado de http://biblioweb.tic.unam.mx/ diccionario/htm/articulos/sec_10.htm.

Anguiano, H. (2003). Historia de la Facultad de Enfermería de la Universidad Autónoma de San Luis Potosí. San Luis Potosí, México: Universidad Autónoma de San Luis Potosí.

Arciga Zavala, B. (2009). Entre la academia y la profesión (mujeres académicas en la Universidad Juárez Autónoma de Tabasco). En L. Romero y N. Esther García (coords.). Voces en diálogo. Construcción de identidades (pp. 133-156). Distrito Federal, México: Plaza y Valdés.

Asociación Nacional de Médicas Mexicanas (2013). Matilde Petra Montoya Lafragua. Recuperado de http://medicasmexicanasac.tripod.com/pag6antecedentes.htm.

Bartra, E. (2001). De las mujeres en la UNAM. En F. Blanco (dir.). Mujeresmexicanas del siglo XX. La otra revolución. Tomo 3 (pp.45-60). Distrito Federal, México: Editorial Edicol.

Bazant, M. (1999). Historia de la educación durante el porfiriato. Distrito Federal, México: El Colegio de México.

Benemérita y Centenaria Escuela Normal del Estado de San Luis Potosí (2013). Historia. Recuperado de http://beceneslp.edu.mx/pagina/?q=historia.

Bolos, S. (2008). Presentación. Espacios públicos/privados: El problema de las mediaciones. En S. Bolos (coord.). Mujeres y espacio público: Construcción y ejercicio de la ciudadanía (pp. 11-28). Distrito Federal, México: Universidad Iberoamericana.

CANo, G. (2000). Género y construcción cultural de las profesiones en el porfiriato: Magisterio, medicina, jurisprudencia y odontología. Historia y Grafía (14): 207243. Recuperado de http://ces.colmex.mx/pdfs/gabriela/g_cano_10.pdf.

Chávez, M. A.; Chávez, M. R., y Ramírez, E. (comp.) (2009). Género y trabajo en las universidades. Guadalajara, México: Instituto Municipal de la Mujeres en Guadalajara, Gobierno Municipal de Guadalajara, Centro Universitario de Ciencias Sociales y Humanidades. Recuperado de http://generomexico.colmex. $\mathrm{mx} /$ textos/LibroGeneroTrabajoUniv.pdf.

De LA Torre, M. (2002). Educación superior en el siglo XX. En L. E. Galván (coord.). Diccionario de la historia de la educación en México. Distrito Federal, México: Universidad Nacional Autónoma de México, Consejo Nacional de Ciencia y 
Tecnología, Centro de Investigaciones y Estudios Superiores en Antropología Social. Recuperado de http://biblioweb.tic.unam.mx/diccionario/htm/articulos/ sec_8.htm.

Delgado, L. (2000). Las primeras tituladas de las diferentes carreras de la UASLP. Universitarios Potosinos (8): 5-13.

Diario Oficial de la Federación (2006). Ley General para la Igualdad entre Mujeres y Hombres. Recuperado de http://dof.gob.mx/nota_detalle.php?codigo $=49267$ $19 \&$ fecha $=02 / 08 / 2006$.

Escamilla, O., y Pineda, H. (2012). Concepción Medizabal, la primera ingeniera mexicana. Gaceta Digital de la Facultad de Ingeniería (5): 9-11. Recuperado de http:// www.ingenieria.unam.mx/paginas/gaceta/2012/gaceta5_2012.pdf.

Estatuto Orgánico de la Universidad Autónoma de San Luis Potosí (1943). Consultado en el Centro de Documentación Histórica Lic. Rafael Montejano y Aguiñaga de la Universidad Autónoma de San Luis Potosí.

Facultad de Ciencias de la UASLP (1998). Titulados en física, matemáticas y electrónica, 1961-1998. San Luis Potosí, México: Universidad Autónoma de San Luis Potosí. Facultad de Estomatología de la UASLP (2007). Memorias de la Facultad de Estomatología, UASLP. San Luis Potosí, México: Universidad Autónoma de San Luis Potosí.

Facultad de Ingeniería de la UASLP (1993). Facultad de Ingeniería. Pasado y presente de la Facultad de Ingeniería. San Luis Potosí, México: Universidad Autónoma de San Luis Potosí.

García Guevara, P. (2004). Mujeres académicas. El caso de una universidad estatal mexicana. Distrito Federal, México: Plaza y Valdés.

Herrera, M. (2011). Rostros del ayer: Dra. María Castro. Boletín Perspectiva. Órgano Informativo de la Secretaría Administrativa de la UASLP (175): 4-9.

Herrera, M. (2012). Rostros del ayer: Q. I. Elisa Leyva Ramos. Boletin Perspectiva. Órgano Informativo de la Secretaría Administrativa de la UASLP (181): 4-13.

Hierro, G. (2007). De la domesticación a la educación de las mexicanas. Distrito Federal, México: Torres Asociados.

IZAGUIRRE, R. (2010). La configuración de la profesión académica. La autonomía en la construcción de la femineidad universitaria. En R. López y V. Félix (coords.). Perfiles culturales de la formación universitaria (pp.133-177). Culiacán, México, Universidad Autónoma de Sinaloa.

El Heraldo (1948, enero 22). La industria potosina sí concurrirá a la exposición de la recuperación: No podía ser de otro modo, ya que San Luis es la tercera ciudad industrial de la República. 
LAGARDE, M. (2003). La cultura feminista hace la diferencia: Claves de género para una gran alternativa. En O. Bustos y N. Blázquez (coords.). Qué dicen las académicas acerca de la UNAM (pp. 111-127). Distrito Federal, México, Universidad Nacional Autónoma de México.

LARA, A. (coord.) (2009). Género en educación. Temas, avances, retos yperspectivas. Distrito Federal, México, Universidad Politécnica Nacional.

Lomnitz, L. (1993). Una familia de la élite mexicana, 1826-1980: Parentesco, clase y cultura. Distrito Federal, México: Alianza.

López, R., y Félix, V. (coords.) (2010). Perfiles culturales de la formación universitaria. Distrito Federal, México: Juan Pablos Editor, Universidad Autónoma de Sinaloa.

Mendieta, L. (1976). Historia de la Facultad de Derecho. Distrito Federal, México: Universidad Nacional Autónoma de México.

Morley, L. (1999). Techo de cristal o jaula de hierro. En M. Belausteguigoitia y A. Mingo (coords.). Géneros prófugos (pp. 349-368). Distrito Federal, México: Universidad Nacional Autónoma de México.

Notimex (2009, octubre 23). Murió la primera alcaldesa interina de México. Recuperado de http://www.wradio.com.mx/noticias/actualidad/murio-la-primera-alcaldesainterina-de-mexico/20091023/nota/899623.aspx.

Paláu, M. T. (2000). Facultad del Hábitat: Historia y perspectivas. San Luis Potosí, México: Universidad Autónoma de San Luis Potosí.

Palermo, A. (2006). El acceso de las mujeres a la educación universitaria. Revista Argentina de Sociologia, 4(7): 11-46. Recuperado de http://redalyc.uaemex.mx/ pdf/269/26940702.pdf.

Pedraza, J. F. (1979). Apuntes históricos de la escuela de Ciencias Quimicas de la Universidad Autónoma de San Luis Potosí. San Luis Potosí, México: Universidad Autónoma de San Luis Potosí.

Peñalosa, J. A. (2000). Chayo Oyárzun, singular mujer potosina. Alumna y maestra de la UASLP. Universitarios Potosinos (43).

Preciado, F. (2006). El tiempo y el espacio de las académicas. Revista de Estudios de Género. La Ventana (24): 151-174. Recuperado de http://www.redalyc.org/articulo. oa?id=88402406.

Reyes, S. (2003). Historia de la Facultad de Contaduría y Administración de la UASLP. San Luis Potosí, México: Universidad Autónoma de San Luis Potosí.

Rivera, A. (2012). Margarita Chorné y Salazar. Recuperado de http://qacontent. edomex.gob.mx/idc/groups/public/documents/edomex_archivo/margaritachorneysalazar.pdf. 
Rivera, J. J., y Montejano y Aguiñaga, R. (1998). La Universidad Autónoma de San Luis Potosi a 75 años de su Autonomía. San Luis Potosí, México: Universidad Autónoma de San Luis Potosí.

Rodríguez de Palacios, A. M. (2001). Dra. Beatriz Velázquez: Su experiencia y reflexiones en el campo de la medicina. Entrevista. Universitarios Potosinos (8): 8-17. Rodríguez, M. A. (2002). Historia de la educación técnica. En L. E. Galván (coord.). Diccionario de la historia de la educación en México. Distrito Federal, México: Universidad Nacional Autónoma de México, Consejo Nacional de Ciencia y Tecnología, Centro de Investigaciones y Estudios Superiores en Antropología Social. Recuperado de http://biblioweb.tic.unam.mx/diccionario/htm/articulos/ sec_14.htm.

Scott, J. (2000). El género: Una categoría útil para el análisis histórico. En M. Lamas (comp.). El género. La construcción cultural de la diferencia sexual (pp. 265-303). Distrito Federal, México: Porrúa.

UASLP (Universidad Autónoma de San Luis Potosí) (2000). UASLP: Algo de su historia. San Luis Potosí, México: Universidad Autónoma de San Luis Potosí.

UASLP (Universidad Autónoma de San Luis Potosí) (2014). Resumen ejecutivo del informe de rectoria 2013-2014. Indicadores institucionales. Recuperado de http://www. uaslp.mx/Documents/Universidad/Sitio\%20rector/Informes/2013-2014/20132014-05-Indicadores-Institucionales.pdf.

Unidad de Enlace de Trasparencia y Acceso a la Información de la UASLP. La UASLP comienza a expedir los títulos en femenino. Expediente 788/TA15.1/003-2015.

Universidad de Colima (2011). II Reunión Nacional de Universidad Públicas e Instituciones de Educación Superior. Caminos para la Equidad de Género. Recuperado de http://201.161.2.34/secciones/convocatorias/pdf/953673Reunion_Nacional.pdf. URRUTIA, E. (coord.) (2005). Estudios sobre las mujeresy las relaciones de género en México: Aportes desde diversas disciplinas. Distrito Federal, México: El Colegio de México. Villegas, J. A. (2007). Apuntes para la historia de la Facultad de Derecho de la UASLP. San Luis Potosí, México: Universidad Autónoma de San Luis Potosí. 